\title{
Estrategias pedagógicas para mitigar la erosión fluvial en la ribera del río Magdalena en Sitionuevo ${ }^{1}$
}

\section{Pedagogical strategies to mitigate fluvial erosion on the banks of the Magdalena River in Sitionuevo}

DOI: http://dx.doi.org/10.17981/cultedusoc.9.3.2018.102

Artículo de investigación. Fecha de recepción: 15/06/2018. Fecha de aceptación: 27/11/2018

\author{
Aida Vivanco-Navarro; León Navarro-Archibold ${ }^{2}$; \\ Gary Nelson De Moya; Rogelia Altamar-Barandica; \\ Gilma Florez-Florez e Immys Gutiérrez ${ }^{3}$ \\ Institución Educativa Departamental San José (Colombia) \\ acriluz2001@hotmail.com
}

Para citar este artículo:

Vivanco-Navarro, A., Navarro-Archibold, L., De Moya, G., Altamar-Barandica, R., Florez-Florez, G. y Gutiérrez, I. (2018). Estrategias pedagógicas para mitigar la erosión fluvial en la ribera del río Magdalena en Sitionuevo. Cultura. Educación y Sociedad 9(3), 861-868. DOI: http://dx.doi.org/10.17981/cultedusoc.9.3.2018.102

\section{Resumen}

La erosión fluvial de la ribera del río Magdalena es un fenómeno que está representando un grave problema sobre la comunidad de Sitionuevo, pues uno de las bases gastronómicas y fuentes nutricionales es el pescado obtenido de tal ribera. Debido a esta problemática surgió el presente estudio con la finalidad de implementar estrategias pedagógicas para mitigar la erosión fluvial en la ribera del río Magdalena en Sitio Nuevo. Metodológicamente se trabajó bajo en enfoque cualitativo desde un alcance descriptivo. Como unidad de análisis se seleccionaron 128 estudiantes de la Institución Educativa Departamental San José. Los resultados dejaron en evidencia que la erosión fluvial en la ribera del río Magdalena tiene un impacto negativo sobre el abastecimiento de peces en la comunidad, donde a través la pedagogía se logró informar a la comunidad para que puedan contribuir a la mitigación de este fenómeno.

Palabras clave: Estrategias pedagógicas, erosión fluvial, Río Magdalena.

\section{Abstract}

The fluvial erosion of the banks of the Magdalena River is a phenomenon that is representing a serious problem on the community of Sitio Nuevo, since one of the gastronomic bases and nutritional sources is the fish obtained from that shore. Due to this problem the present study arose in order to implement pedagogical strategies to mitigate river erosion on the banks of the Magdalena river in $\mathrm{Si}$ tio Nuevo. Methodologically, work was carried out under the perspective of research as a pedagogical strategy. As a unit of analysis, 128 students from the Departmental Educational Institution San José were selected. The results showed that fluvial erosion on the banks of the Magdalena River has a negative impact on the fish supply in the community, where through pedagogy the community was informed so that they can contribute to the mitigation of this phenomenon.

Keywords: Pedagogical strategies, fluvial erosión, Magdalena River.

1 Este artículo ha sido derivado del Programa de Fortalecimiento de la Cultura Ciudadana y Democrática CT+I a través de la IEP apoyada en TIC en el Departamento de Magdalena: CICLON

2 Líder del grupo de investigación: Grupo de investigación "Los coquitos".

3 Docentes de la Institución Educativa Departamental San José y del grupo de investigación "Los coquitos"

- The author; licensee Universidad de la Costa - CUC.

Cultura, Educación y Sociedad vol. 9 no. 3, pp. 861-868. Diciembre, 2018

Barranquilla. ISSN 2389-7724 Online 


\section{Introducción}

La educación ambiental es un elemento utilizado desde las escuelas para crear conciencia en la comunidad educativa, ésta busca no solamente identificar un problema, sino encontrar una solución para prevenir el deterioro ambiental por medio de la integración de los saberes, actitudes y actos, comprendiendo de este modo el problema y emprendiendo acciones para mitigar dicho deterioro. Es decir, la educación ambiental busca una conducta responsable, conocimientos sobre el medio ambiente y su problemática, además de estrategias para actuar sobre y para el medio (Navarro y Garrido, 2006).

Existen nuevas estrategias educativas para entender y mitigar, desde diversos puntos de vista, el deterioro ambiental actual. La educación ambiental (EA) es una herramienta para despertar interés en las personas sobre la relevancia del cuidado de su entorno, así mismo, busca incrementar los conocimientos, y con estos impulsar a la modificación de conductas, valores y estilo de vida, con el objetivo de la disminuir y prevenir problemas existentes y futuros. La (EA) se encamina a la acción, donde se debe incluir a los estudiantes en la solución de los problemas reales y específicos, no solo con el fin de plantear posibles soluciones sino llevarlas a cabo y obtener capacidades generales, habilidades y rutinas concretas mediante estrategias de mejora del medio. (Espejel \& Flores, 2012).

La educación a lo largo del tiempo ha presentado cambios significativos, que invitan al docente a diseñar e implementar nuevas estrategias pedagógicas para dirigir el proceso de enseñanza con sus estudiantes, una estrategia pedagógica es un conjunto de actividades que se realizan con un orden lógico y coherente en función del cumplimiento de objetivos del plan académico. Es decir, es una planificación que contiene métodos o acciones que permiten al estudiante alcanzar los logros propuestos, mejorar su aprendizaje y facilitar su crecimiento personal (Picardo, Balmore, \& Escobar, 2004).

En ese sentido, cuando se concibe desde unos enfoques educativos críticos la investigación, se manifiesta un desafío teórico-práctico por la manera como sus estimaciones sobre el saber, la ciencia, su epistemología, la cultura, lo humano y los grupos sociales que durante cuatro siglos ajustan acciones que han sido reconocidas por su objetividad, y la forma no solo de ella misma (Mejía, Manjarrés, 2010).

De esta forma, la investigación es percibida como una estrategia pedagógica que conlleva: Generar momentos de inclusión, en donde la heterogeneidad es aceptada y respetada; además, de que permite poner en manifiesto los conductos de creatividad del docente, y transformar los diferentes interrogantes que se presentan por parte de los grupos de investigación de aprendizaje colaborativo, constituidos por niñas, niños y adolescentes. Asimismo, conduce a los estudiantes a tomar un rol activo en el proceso de aprendizaje mediante la construcción de su propio conocimiento, potenciación de habilidades y destrezas, convirtiendo la educación en un canal de formación, entretenimiento y oportunidades. (Camargo, 2015).

Por su parte Según diversos autores las tecnologías de la información y la comunicación, permiten desarrollar competencias sociales, que incluso se ha utilizado para trabajar en instituciones educativas con deterioro de la convivencia escolar como factores asociados al desempeño en estudiantes de básica primaria con experiencia de desplazamiento forzado (Avendaño, Cortés, Guerrero, 2015).

La principal, fuente de abastecimiento de múltiples departamentos de la región caribe en colombia es el rìo magdalena, lo que convierte los problemas del río en problemas de todo el país. (Ángel, 2005) En los últimos años se ha evidenciado un problema por erosión fluvial en la ribera del río 
magdalena en el municipio de Sitionuevo, Magdalena, afectando en gran manera la labor pesquera que se lleva a cabo en este lugar y ocasionando problemas con la principal fuente nutricional de la comunidad. (Restrepo, 2005).

La erosión fluvial, puede ser definida como el acto de deterioro producido por las aguas fuertes y de torrentes. la acción de desgaste ocasionado por las aguas de torrentes, aguas salvajes, y ríos. (Solanes, 1996). al terminar las fuertes lluvias se forman las llamadas lagunas de corriente, formando un canal de feroces aguas de forma disforme;su acción es destructiva, al igual que la de las aguas salvajes, (Schreider, 2015). los ríos se caracterizan por erosionar verticalmente el terreno; la mayor prueba de ello son los valles fluviales y los cañones. (Bernal, 1996).

Las aguas continentales hacen parte de una primera etapa en que la erosión mecánica provocada por el agua (Herrera-Mendoza y Morales, 2013) En forma de ríos que discurren de corrientes subterráneas o sobre la superficie, el agua cumple la función de desgastar a su paso todos los materiales arrastrandolos por último hacia el mar, es decir, que estos terminan formando el nuevo paisaje cuando llegan a los nuevos suelos. (Martínez, 2005). las cascadas desfiladeros y grutas, son conformadas por agua que en ocasiones terminan inundando cierta regiones, aunque a los márgenes de de los ríos la vida se ha desarrollado en mayor cantidad. (Vide, 2007).

Por otra parte, la erosión debida a las aguas corrientes sigue las mismas etapas en que se divide de forma natural el curso de un río. en la primera etapa en que la erosión mecánica provocada por el agua y los materiales que acarrea es muy fuerte en el tramo más alto del río. (Restrepo, 2005). En la segunda etapa de transporte, la erosión mecánica no se detiene, pero inicia una erosión química. Esta se presenta en el tramo medio del río. Finalmente, en el tramo bajo se antepone una concentración de los materiales transportados, llevando a que la acción mecánica se reduzca en gran medida y prácticamente sólo actúe la erosión química. (Ángel, 2005).

Además, la acción erosiva de un río es a causa de que la energía del agua puede arrancar trozos de roca que no solamente son llevados por la corriente, sino que además, actúan como un martillo sobre el canal del río, arrancando nuevos fragmentos (Herrera-Mendoza, Acuña, Ramírez y De la Hoz, 2016), debido a la irregularidad que se da en el cauce del río, es común la formación de remolinos que no solo arrastran arenas y gravas, sino que también, pulen su fondo y crean cavidades. Otras veces, la elevada pendiente crea saltos, cascadas o cataratas, de las cuales algunas llegan hasta los 1000 metros de altura (Ortega, 2008). En otros casos, cuando el canal se encuentra con grandes obstáculos, el agua explora los lugares más frágiles para luego desgastarlos y formar desfiladeros o cañones (Restrepo, 2015). Son más frecuentes las cuevas subterráneas en terrenos calcáreos causadas por la erosión química del agua, que transforma el carbonato insoluble en bicarbonato soluble (Rodríguez, 2005).

El caudal de un río puede sobrepasar su cauce cuando crecen excesivamente debido a las temporadas de lluvias o el deshielo, desbordando hacia las riberas. suele producirse de forma gradual este desbordamiento, pero otras lo hacen de manera abrupta, provocando una gran erosión en todo el terreno (Crissien-Borrero, Cortés-Peña y HerreraMendoza, 2016).

El resultado de la erosión se basa en materiales más o menos finos que arrastrados por el río durante todo el camino. En el curso medio comienzan a sedimentarse cuando la fuerza de la corriente no puede mantener estas partículas en suspensión (Bernal, 1996). Pero la fuerza erosiva actúa después sobre estos depósitos y los desgasta más por la zona en que la velocidad del agua 
es mayor, mientras deposita nuevos materiales donde es más débil. (Lorenc, 1995). El resultado final son unos depósitos de forma sinuosa que llamamos meandros. el río puede volver a abrirse paso en línea recta con el tiempo y las crecidas, dejando en sus márgenes lagunas en forma de media luna (Ángel, 2005). Donde el final del proceso erosivo fluvial tiene lugar en la desembocadura del río, en algunos casos la fuerza de la corriente es capaz de seguir erosionando el fondo de la plataforma continental y formar un valle submarino. (Sepúlveda, 2009).

Desde el punto de vista de los procesos fluviales, la morfología fluvial es el resultado de una compleja interacción entre los procesos erosivos y de depósito. Las relaciones entre estos diferentes procesos configuran las características generales del sistema fluvial (Howard, 1994). Por otro lado, los sedimentos son transportados y depositados en sistemas fluviales por tres principales mecanismos, carga de fondo y carga en suspensión (Kokot, 2004).

Continuando con este orden de ideas, aparecen los flujos de detritos, estos son flujos plásticos, ricos en sedimentos donde la cantidad de agua existente en los poros es baja (López, 2008). El flujo ocurre cuando una gran cantidad de sedimentos es movilizada por licuefacción e una superficie inclinada. El movimiento termina cuando el flujo pierde momento o cuando el agua existente en los poros escapa del sedimento cesando la lubricación de los granos. (Herbas, 2006), (Osejo, 1997). En algunos casos, como causa de la disminución de la viscosidad a través de la dilución, los flujos de detritos pueden volverse turbulentos, formando flujos hiperconcentrados. Los depósitos generados son pobremente seleccionados, compuestos por clastos que pueden variar de tamaño de gránulos hasta bloques, separados por una matriz areno-silítica-arcillosa. A veces se puede observar una orientación incipiente de los clastos. (Samaniego, 2008).

\section{Metodología}

\section{Diseño}

Se realizó un estudio de tipo cualitativo, desde un diseño descriptivo. La investigación cualitativa busca conocer e interpretar la realidad de los participantes a través de sus propias experiencias, entregando una información subjetiva del fenómeno de estudio. Donde se buscó generar una producción de conocimiento basado en la reflexión de los participantes, teniendo en cuenta la participación activa de maestros en el proceso de enseñanza y acompañamiento tecnológico, guiando al estudiante en su proceso de investigación. Generando nuevo saber y conocimiento sobre una realidad determinada en un proceso de aprendizaje colaborativo.

Donde los estudios con un diseño descriptivo están encaminados a especificar las características de la población sujeto de estudio o los fenómenos que sean sometidos a análisis. Bajo un estudio de tipo exploratorio, debido a que no se han realizado estudios previos en la institución. (Hernández, Fernández, y Baptista, 2010).

\section{Participantes}

Durante el estudio participaron 128 estudiantes distribuidos en los cursos pertenecientes a la educación básica secundaria, con edades que oscilaron entre los 13 y 16 años, de la Institución Educativa Departamental San José, del Municipio de Sitio Nuevo, Magdalena.

\section{Técnicas e instrumentos}

Se seleccionaron técnicas e instrumentos de recolección de información propios de la investigación cualitativa, ente ellos la observación participante y la entrevista en profundidad. 


\section{Procedimiento}

El procedimiento se diseñó a partir de los recorridos de las trayectorias de indagación, basados en la integración de la IEP apoyada en TIC al aula, articulado al plan de estudios, para el cual se plantearon cuatro fases: Fase 1: Planteamiento del problema. Fase 2: Selección, diseño y aplicación de técnicas de recolección de datos.

Fase 3: Sistematización de los resultados. Fase 4: Apropiación social del saber y conocimiento.

\section{Resultados}

A continuación, se presentan los principales hallazgos del estudio partiendo de las fases ejecutadas en el proceso de implementación, con la finalidad de generar estrategias pedagógicas para mitigar la erosión fluvial en la ribera del rio Magdalena en el Municipio de Sitio Nuevo, (Ver tabla 1)

TABLA 1

Sistematización de los resultados obtenidos en las fases de implementación del estudio

Fase

Planteamiento del problema.

Selección, diseño y aplicación de técnicas de recolección de datos.

Sistematización de los resultados.

Apropiación social del saber y conocimiento.
Resultado

Los estudiantes, con el acompañamiento de maestros y maestras identificaron el problema y realizaron el proceso de indagación, donde se realizó la revisión literaria. En este sentido fue posible establecer que existen dificultades en la manera en la que los individuos del Municipio se relacionan con las problemáticas medioambientales. En el caso de la Ribera del Rio Magdalena, se estableció como problema la contaminación existente entre los habitantes, que conduce a la inundación y deterioro del ecosistema. Tal situación dificulta las actividades económicas de los pescadores, la vida de quienes se encuentran en sectores aledaños

Los estudiantes estructuraron entrevistas, que fueron realizadas a los pescadores y personas que viven en los sitios aledaños a la ribera, que han observado la erosión fluvial que se viene presentando desde hace unos años.

Se realizó el análisis de la información donde se logró articular las experiencias de la comunidad con la teoría encontrada mediante el proceso de indagación.

Los estudiantes con el acompañamiento de los maestros y maestras presentaron sus resultados a la comunidad en una feria institucional, donde expusieron las diferentes estrategias y comportamientos proambientales que fueron resultado de la sistematización de los resultados y la revisión teórica, para generar en la comunidad conductas que ayudaran a mitigar la erosión fluvial en la ribera del Rio Magdalena. 
Promover la mitigación de la erosión fluvial en la ribera del río Magdalena en Sitionuevo, fue necesaria la intervención de dos fases durante el mismo. Primero se realizó una serie de encuestas a los pescadores y personas que viven en sitios aledaños a la ribera y fueran testigos de la erosión fluvial que se viene presentando desde hace unos años, posteriormente se procedió a la fase pedagógica mediante la cual se consiguió concientizar a los habitantes de Sitionuevo sobre el impacto negativo que estamos generando en el río Magdalena, fuente de recursos hídricos de la población y de gran parte de la Región Caribe (Herrera, 2011).

Con la información adquirida se deduce que la erosión fluvial en Sitionuevo y su marcada aceleración en las dos últimas décadas es el resultado de la contaminación ambiental que ha tocado fuertemente a lo largo del río Magdalena. Está a su vez causa el desbordamiento de caños, ciénagas y otros, con ello se genera el desprendimiento de material arenoso que es arrastrado por las mismas corrientes y luego sucede la sedimentación, que es el proceso de acumulación del material arenoso en otros lugares del río.

Con la sedimentación del río, en Sitionuevo, se han formado islas que influyen directamente sobre el cauce, cambiando la dirección natural de las corrientes que provocan el choque con la ribera aumentándose notablemente la erosión en esta zona. Como consecuencia se tiene la gran pérdida de tierras medidas en hectáreas.

\section{Discusión}

Los resultados anteriormente se contrastan con la idea que, la educación ambiental es un elemento utilizado desde las escuelas para crear consciencia en la comunidad educativa, está debe integrar conocimientos, actitudes y acciones, donde no sólo se informe sobre un determinado problema sino además encontrar respuesta o soluciones para detener y evitar el deterioro ambiental; aún más, se trata de asumir, comprender el problema y actuar sobre él. Es decir, la educación ambiental busca una conducta responsable, conocimientos sobre el medio ambiente y su problemática, además de estrategias para actuar sobre y para el medio (Navarro y Garrido, 2006).

Observando que utilizar estrategias pedagógicas basadas en investigación permite realizar intervenciones para reducir el impacto negativo de la erosión fluvial sobre la población, lo que se logró mediante este proyecto gracias a la fase pedagógica del mismo en el cual se informó a la comunidad sobre los impactos que este fenómeno trae sobre la población y la pesca, y cómo ellos pueden contribuir a la mitigación de esta problemática que se está presentando desde hace unas décadas en la comunidad de Sitionuevo, Magdalena, lo cual se sustenta según los planteamientos de Ortega, Peñuela \& López, (2009). Quienes plantean que es necesario pensar la investigación desde las corrientes educativas críticas, lo que significa crear una lucha teórico-práctica por la manera como sus presupuestos sobre el conocimiento, la ciencia, su epistemología, la cultura, lo humano y los grupos sociales enmarcan una acción que durante cuatrocientos años ha sido señalada como objetiva, y que no solo ha construido una forma de ella, sino que también ha ayudado a generar formas de poder que en la sociedad han servido para el control y la gestación de desigualdades, y en estos tiempos, nuevas formas de acumulación y dominación.

\section{Referencias}

Ángel, J. (2005). Los sistemas hídricos fluviales: visión general.Los sedimentos del río Magdalena: reflejo de la crisis ambiental, 21. 
Avendaño, I., Cortés, O., \& Guerrero, H, (2015). Competencias sociales y tecnologías de la información y la comunicación como factores asociados al desempeño en estudiantes de básica primaria con experiencia de desplazamiento forzado. Divers: Perspect. Psicol. Vol. 11. $N^{o} 1$.

Bernal, G. (1996). Caracterización geomorfológica de la Llanura Deltaica del Río Magdalena, con énfasis en el sistema lagunar de la Ciénaga Grande de Santa Marta, Colombia. Bol. Invest. Mar. Cost, $25,19-48$.

Camargo, C. A. (2015). "La investigación como estrategia pedagógica en la Guajira, desde una perspectiva de la inclusividad y diversidad como nuevo paradigma para el desarrollo de habilidades, destrezas y competencias". Educación y ciudad, (29), 149-162.

Crissien-Borrero, T., Cortés-Peña, O., \& Herrera-Mendoza, K. (2016). Pro-Environmental Assessment and Sustainable Consumption of Household Public Services in Barranquilla Colombia. The European Proceedings of Social \& Behavioural Sciences - EpSBS, 17, 434440. $\quad$ http://dx.doi.org/10.15405/ epsbs.2016.11

Cornelio, L. (2009). Influencia de tres tipos de vegetación ribereña en el control de la erosión fluvial en Pantanos de Centla, México. Revista de Biología Tropical, 57(4), 1153-1163.

Cuervo, G. (2008). DINÁMICA DE CUERPOS DE AGUA Y ZONAS INUNDABLES. Río Magdalena: Navegación Marítima y Fluvial (1986-2008). Barranquilla: Uninorte.

Espejel, A., \& Flores, A. (2012). Educación ambiental escolar y comunitaria en el nivel medio superior, Puebla-Tlaxcala, México. Revista mexicana de investigación educativa.
Herrera, K. (2011). La crisis medioambiental. Una mirada desde la psicología. Ed. Educosta: Colombia.

Herrera-Mendoza, K., Acuña, M., Ramírez, M., y De la Hoz, M. (2016). Actitud y conducta proecológica en jóvenes universitarios. Revista opción. Vol. 32(13). Pp. 456-477.

Herrera, K., y Morales, E. (2013). Perspectiva de la ecología en la comprensión de los comportamientos ambientales. Revista Omnia. Vol. 19(3): pp. 20-30.

Hernández, R., Fernández, C., \& Baptista, P. (2010). Metodología de la investigación. Editorial Mc Graw Hill. México.

Howard, A., Dietrich, W., \& Seidl, M. (1994). Modeling fluvial erosion on regional to continental scales. Journal of Geophysical Research: Solid Earth, 99(B7), 13971-13986.

Kokot, R. (2004). Erosión en la costa patagónica por cambio climático. Revista de la Asociación Geológica Argentina, 59(4), 715-726.

Lorenc, M. W., Muñoz Barco, P., \& Saavedra, J. (1995). Marmitas de gigante en el valle del río Jerte como ejemplo de erosión fluvial intensiva por remolinos e influencia tectónica en su distribución y morfología. Cuaternario y Geomorfología, 9(1/2), 17-26.

López, M. (2008). El control del río Colorado como factor histórico La necesidad de estudiar la relación tierra/agua. Frontera Norte, 20(40), 49-78.

Martínez, J. I., \& López, E. (2005). El sistema fluvial del Magdalena durante el Plioceno-Cuaternario: Contexto tectónico y escenario paleoclimático. Los sedimentos del Río Magdalena: Reflejo de la crisis ambiental, 101-112.

Mejía,M. Manjarrés, M. (2010). "La investigación como estrategia pedagógica" Programa de ondas-colciencias 
Navarro, R. y Garrido, S. (2006). "Construyendo el significado del cuidado ambiental: un estudio de caso en educación secundaria", Reice. Revista electrónica iberoamericana sobre calidad eficacia y cambio en educación (España), vol. 4, núm. 1, pp. 52-70.

Ortega, M. A. (Ed.). (2008). Río Magdalena, navegación marítima y fluvial (1986-2008). Universidad del Norte.

Osejo, A. Á. (1997). Gestión del Agua Subterránea en la Sabana de Bogotá. Geología Colombiana, 22, 81-101.

Picardo, O., Balmore, R., \& Escobar, J. (2004). Diccionario enciclopédico de ciencias de la educación. San Salvador: El Salvador.

Restrepo, A., \& Juan, D. (2015). The impact of deforestation on the erosion in the Magdalena River drainage basin (1980-2010). Revista de la Academia Colombiana de Ciencias Exactas, Físicas y Naturales, 39(151), 250-267.
Restrepo, J. C., \& Restrepo, J. D. (2005). Efectos naturales y antrópicos en la producción de sedimentos de la cuenca del río Magdalena. Revista de la Academia Colombiana de Ciencias Exactas, Físicas y Naturales, 29(111), 239-254.

Rodríguez, N., \& Armenteras, D. (2005). Ecosistemas naturales de la cuenca del rio Magdalena. Los sedimentos del río Magdalena: reflejo de la crisis ambiental, 79-98.

Samaniego, M. (2008). El control del río Colorado como factor histórico: La necesidad de estudiar la relación tierra/ agua. Frontera norte, 20(40), 49-78.

Sepúlveda, A., Geissen, V., Ochoa, S., Jarquín, A., Hernández, S., Capetillo, E., \& Zamora- Schreider, M., Scacchi, G., Franco, F., Fuentes, R., \& Moreno, C. (2015). Aplicación del método de Lischtvan y Lebediev al cálculo de la erosión general. Tecnología y Ciencias del Agua, 16(1), 15-26.

Solanes, M. (1996). Mercados de derechos de agua: componentes institucionales. Revista de la CEPAL.

Vide, J. P. M. (2007). Ingeniería de ríos (Vol. 9). Univ. Politèc. de Catalunya. 